\title{
GENERALIZATIONS OF THE STANDARD ARTIN REPRESENTATION ARE UNITARY
}

\author{
MOHAMMAD N. ABDULRAHIM
}

Received 27 October 2004 and in revised form 24 February 2005

We consider the Magnus representation of the image of the braid group under the generalizations of the standard Artin representation discovered by M. Wada. We show that the images of the generators of the braid group under the Magnus representation are unitary relative to a Hermitian matrix. As a special case, we get that the Burau representation is unitary, which was known and proved by C. C. Squier.

\section{Introduction}

The braid group $B_{n}$ has a well-known representation due to $\operatorname{Artin}$ in the group $\operatorname{Aut}\left(F_{n}\right)$ of automorphisms of the free group $F_{n}$ generated by $x_{1}, \ldots, x_{n}$. The automorphism corresponding to the braid generator $\sigma_{i}$ takes $x_{i}$ to $x_{i} x_{i+1} x_{i}^{-1} ; x_{i+1}$ to $x_{i}$, and fixes all other free generators. Such a representation of the braid group by automorphisms of a free group was proved to be faithful [3, page 25].

In Section 2, we present an infinite series of representations generalizing the standard Artin representation, which were discovered by Wada [8]. More precisely, for an arbitrary nonzero integer $k$, the automorphism corresponding to the braid generator $\sigma_{i}$ takes $x_{i}$ to $x_{i}{ }^{k} x_{i+1} x_{i}{ }^{-k} ; x_{i+1}$ to $x_{i}$, and fixes all other free generators. Shpilrain has shown that these representations are indeed faithful [6, page 773].

In Section 3, after having defined the automorphism corresponding to the braid generator, suggested by Wada, we apply the Magnus representation to these subgroups of $\operatorname{Aut}\left(F_{n}\right)$ to get linear irreducible representations $B_{n} \rightarrow \mathrm{GL}_{n-1}\left(\mathbb{C}\left[t^{ \pm 1}\right]\right)$. We show that for any nonzero integer $k$, the linear representations obtained are unitary relative to a Hermitian matrix. In particular, this shows that the Burau representation, namely when $k=1$, is conjugate to an ordinary unitary representation; which was proved by Squier [7].

Showing that Wada's representations are unitary might possibly help us to determine whether or not such matrix representations of the braid group are faithful. A similar argument was done in the case of the standard Artin representation (see [1, page 1257]). It was known that for $k=1$, the Burau representation is not faithful for $n \geq 6$ [5]. It is now known that the Burau representation for $n=5$ is not faithful [2]. 


\section{Generalizations of the standard Artin representation}

\section{Definitions}

The braid group on $n$ strings, $B_{n}$, is the abstract group with generators $\sigma_{1}, \ldots, \sigma_{n-1}$ and a presentation as follows:

$$
\begin{gathered}
\sigma_{i} \sigma_{i+1} \sigma_{i}=\sigma_{i+1} \sigma_{i} \sigma_{i+1}, \quad i=1,2, \ldots, n-2, \\
\sigma_{i} \sigma_{j}=\sigma_{j} \sigma_{i}, \quad|i-j| \geq 2 .
\end{gathered}
$$

According to the standard Artin representation, the automorphism corresponding to $\sigma_{i}$ sends $x_{i}$ to $x_{i} x_{i+1} x_{i}{ }^{-1} ; x_{i+1}$ to $x_{i}$, and fixes all other free generators.

Definition 2.1. The generalizations of the standard Artin representation, discovered by Wada, assert that the automorphism corresponding to $\sigma_{i}$ takes

$$
\begin{gathered}
x_{i} \longrightarrow x_{i}{ }^{k} x_{i+1} x_{i}{ }^{-k}, \\
x_{i+1} \longrightarrow x_{i}, \\
x_{j} \longrightarrow x_{j} \text { for } j \neq i, i+1 .
\end{gathered}
$$

By applying the Magnus representation to the image of the braid group under the generalization of the standard Artin representation, we determine the linear representations $B_{n} \rightarrow \mathrm{GL}_{n}\left(\mathbb{C}\left[t^{ \pm 1}\right]\right)$ [3]. The automorphism $\sigma_{i}$ is mapped onto the $n \times n$ matrix which differs from the identity only by a $2 \times 2$ block with the top-left corner in the $(i, i)$ th place. More precisely,

$$
\sigma_{i}(t)=\left(\begin{array}{c|rr|c}
I_{i-1} & \multicolumn{2}{|c|}{0} & 0 \\
\hline 0 & 1-t^{k} & t^{k} & 0 \\
\hline 0 & 1 & 0 & 0
\end{array}\right) \quad \text { for } i=1,2, \ldots, n-1 .
$$

It is clear that the subspace generated by the column vector $(1,1, \ldots, 1)^{T}$ is invariant under this representation, where $T$ is the transpose. Therefore, these representations, for different values of $k$, are reducible.

Definition 2.2. Let $k \in \mathbb{Z}-\{0\}$. Wada's representations $\left\{\phi_{k}\right\}: B_{n} \rightarrow \mathrm{GL}_{n-1}\left(\mathbb{C}\left[t^{ \pm 1}\right]\right)$ are a family of linear irreducible representations defined as $\phi_{k}\left(\sigma_{i}\right)=I_{n-1}-A_{i} B_{i}$, where

$$
\begin{gathered}
A_{1}=(t^{k}+1,-1, \underbrace{0, \ldots, 0}_{n-3})^{T}, \quad A_{i}=(\underbrace{0, \ldots, 0}_{i-2},-t^{k}, t^{k}+1,-1, \underbrace{0, \ldots, 0}_{n-i-2})^{T}, \\
A_{n-1}=(\underbrace{0, \ldots, 0}_{n-3},-t^{k}, t^{k}+1)^{T},
\end{gathered}
$$

for $i=2, \ldots, n-2$.

Here, $\left\{B_{1}, \ldots, B_{n-1}\right\}$ is the standard basis of $\mathbb{C}^{n-1}$. 
These representations are irreducible by [4, Theorem 5]. Notice that the representation $\phi_{1}$ is (conjugate to) the reduced Burau representation of the braid group as presented in [4].

\section{Wada's representations are unitary}

Notation 3.1. Let $(*): M_{m}\left(\mathbb{C}\left[t^{ \pm 1}\right]\right)$ be an involution defined as follows:

$$
\left(f_{i j}(t)\right)^{*}=f_{j i}\left(t^{-1}\right), \quad f_{i j}(t) \in \mathbb{C}\left[t^{ \pm 1}\right] .
$$

Definition 3.2. Let $X$ and $U$ be elements of $\mathrm{GL}_{n-1}\left(\mathbb{C}\left[t^{ \pm 1}\right]\right)$. $U$ is called a unitary element (relative to $X$ ) if $U X U^{*}=X$.

Now define the following $(n-1) \times(n-1)$ matrix, $M$, in a way that each column looks like $\left(0, \ldots, 0,-t^{k}, t^{k}+1,-1,0, \ldots, 0\right)^{T}$, where $t^{k}+1$ is a diagonal entry and $T$ is the transpose. More precisely, we have

$$
M=\left(\begin{array}{cccccc}
t^{k}+1 & -t^{k} & 0 & \cdots & \cdots & 0 \\
-1 & t^{k}+1 & -t^{k} & 0 & \cdots & \vdots \\
0 & -1 & t^{k}+1 & -t^{k} & \cdots & \vdots \\
0 & 0 & -1 & \ddots & \ddots & 0 \\
\vdots & \vdots & \ldots & 0 & t^{k}+1 & -t^{k} \\
0 & 0 & \cdots & 0 & -1 & t^{k}+1
\end{array}\right) .
$$

For simplicity, we denote the matrix $\phi_{k}\left(\sigma_{i}\right)$ corresponding to the braid generator, $\sigma_{i}$, under Wada's representations, by $X_{k, i}$, where $X_{k, i}=I_{n-1}-A_{i} B_{i}$, where $A_{i}, B_{i}$ are given by Definition 2.2.

We now prove our main theorem.

TheOREm 3.3. The images of the generators of $B_{n}$ under Wada's representations, $\phi_{k}$, are unitary relative to $M$, that is, for $1 \leq i \leq n-1$,

$$
X_{k, i} M\left(X_{k, i}\right)^{*}=M .
$$

Proof.

$$
\begin{aligned}
X_{k, i} M\left(X_{k, i}\right)^{*} & =\left(I-A_{i} B_{i}\right) M\left(I-A_{i} B_{i}\right)^{*} \\
& =M-A_{i} B_{i} M-M B_{i}{ }^{*} A_{i}{ }^{*}+A_{i} B_{i} M B_{i}{ }^{*} A_{i}{ }^{*} .
\end{aligned}
$$

Having done some computations, we get

$$
\begin{gathered}
A_{i} B_{i} M=t^{k} A_{i} A_{i}{ }^{*}, \\
M B_{i}{ }^{*} A_{i}{ }^{*}=A_{i} A_{i}{ }^{*}, \\
A_{i} B_{i} M B_{i}{ }^{*} A_{i}{ }^{*}=\left(t^{k}+1\right) A_{i} A_{i}{ }^{*} .
\end{gathered}
$$


So,

$$
X_{k, i} M\left(X_{k, i}\right)^{*}=M+A_{i} A_{i}^{*}\left(-t^{k}-1+t^{k}+1\right)=M \text {. }
$$

Now we view $\mathbb{C}\left[t^{ \pm 1}\right]$ as a subring of $\mathbb{C}\left[u, u^{-1}\right]$, where $u^{2}=t$. Let $N=u^{-k} M$, then by direct substitution, we get

$$
N=\left(\begin{array}{cccccc}
u^{k}+u^{-k} & -u^{k} & 0 & \cdots & \cdots & 0 \\
-u^{-k} & u^{k}+u^{-k} & -u^{k} & 0 & \cdots & \vdots \\
0 & -u^{-k} & u^{k}+u^{-k} & -u^{k} & \cdots & \vdots \\
0 & 0 & -u^{-k} & \ddots & \ddots & 0 \\
\vdots & \vdots & \cdots & 0 & u^{k}+u^{-k} & -u^{k} \\
0 & 0 & \cdots & 0 & -u^{-k} & u^{k}+u^{-k}
\end{array}\right)
$$

It is clear that $N$ is Hermitian $\left(N^{*}=N\right)$ and $X_{k, i} N\left(X_{k, i}\right)^{*}=N$. Next, our objective is to show that a certain specialization $\bar{N}$ of $N$ is equivalent to the identity matrix in some extension field, that is, for some matrix $U$, we have that

$$
\bar{N}=U U^{*}
$$

From linear algebra, it is well known that a Hermitian matrix is positive definite if and only if each of the principal minors is positive. In that case, the matrix will be equivalent to the identity matrix.

The principal minors of $N$ are of the form $\operatorname{det}\left(D_{m}\right)$, where $1 \leq m \leq n-1$ and $D_{m}$ is an $m \times m$ matrix (upper-left corners of $N$ ). It is then easy to see the following lemma.

Lemma 3.4. Let $t=u^{2}$ and $u=1$, then under this specialization, for $1 \leq m \leq n-1$,

$$
\operatorname{det}\left(D_{m}\right)=m+1
$$

Proof. By induction on $m$, we get

$$
\operatorname{det}\left(D_{m}\right)=\frac{u^{2(m+1) k}-1}{u^{m k}\left(u^{2 k}-1\right)}=u^{-m k}\left(u^{2 m k}+u^{2(m-1) k}+\cdots+u^{2 k}+1\right) .
$$

Having $u=1$, we get that $\operatorname{det}\left(D_{m}\right)=m+1$.

Let $u=a$, where $a$ is a complex number lying in an open arc around 1 on the unit circle. By having an explicit formula for the principal minors of $N$ as in Lemma 3.4, it is then possible to completely determine the arc around 1 where $a$ belongs to. The choice of this arc depends on the values of $k$ and $n$. Along the same lines as in [1, pages 1254-1255], we can easily get the following lemma. 
Lemma 3.5. Let a be a complex number on the unit circle. Then $\operatorname{det}\left(D_{m}\right)$ is positive for all $m=1,2, \ldots, n-1$ if and only if a lies in an open arc around 1 bounded by $e^{-\pi i / k n}$ and $e^{\pi i / k n}$.

Hence, the matrix $N$ is a positive definite Hermitian matrix under the complex specialization $u=a$ belonging to the open arc bounded by $e^{-\pi i / k n}$ and $e^{\pi i / k n}$. We denote this matrix by $\bar{N}$. By a theorem in linear algebra, there exists a matrix $U$ such that

$$
\bar{N}=U U^{*} .
$$

As in [1, page 1255], the next theorem shows that a conjugate of Wada's representation is unitary. Here, a matrix $X$ is unitary if $X X^{*}=X^{*} X=I$.

Theorem 3.6. The complex specialization of Wada's representation of $B_{n}$ (having $t=u^{2}=$ $a^{2}$ and $a$ is around 1 ) is conjugate to an ordinary unitary representation.

Proof. Consider the composition map

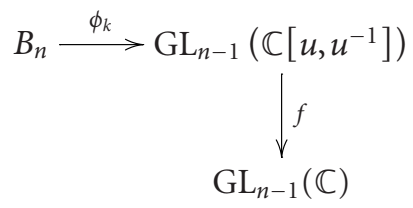

Let $f\left(X_{k, i}\right)$ be the image of $X_{k, i}$ under the complex specialization $u=a$, where $a$ lies in an arc around 1 bounded by $e^{-\pi i / k n}$ and $e^{\pi i / k n}$.

Having that $\bar{N}=U U^{*}$, we let $V=U^{-1} f\left(X_{k, i}\right) U$, then it is clear that

$$
V V^{*}=V^{*} V=I
$$

Notice that, under the case $k=1$, Theorem 3.6 implies that the specialization of the Burau representation is conjugate to an ordinary unitary representation; which was proved by Squier [7].

\section{Acknowledgment}

This note is in final form and no version of it will be submitted for publication elsewhere.

\section{References}

[1] M. N. Abdulrahim, A faithfulness criterion for the Gassner representation of the pure braid group, Proc. Amer. Math. Soc. 125 (1997), no. 5, 1249-1257.

[2] S. Bigelow, The Burau representation is not faithful for $n=5$, Geom. Topol. 3 (1999), 397-404.

[3] J. S. Birman, Erratum: "Braids, Links, and Mapping Class Groups," (Ann. of Math. Studies, no. 82, Princeton University Press, New Jersey, 1974), Princeton University Press, New Jersey, 1975.

[4] E. Formanek, Braid group representations of low degree, Proc. London Math. Soc. 73 (1996), no. 3, 279-322.

[5] D. D. Long and M. Paton, The Burau representation is not faithful for $n \geqslant 6$, Topology 32 (1993), no. $2,439-447$. 


\section{Generalizations of the standard Artin representation}

[6] V. Shpilrain, Representing braids by automorphisms, Internat. J. Algebra Comput. 11 (2001), no. $6,773-777$.

[7] C. C. Squier, The Burau representation is unitary, Proc. Amer. Math. Soc. 90 (1984), no. 2, 199-202.

[8] M. Wada, Group invariants of links, Topology 31 (1992), no. 2, 399-406.

Mohammad N. Abdulrahim: Department of Mathematics, Faculty of Science, Beirut Arab University, P.O. Box 11-5020, Beirut 1107 2809, Lebanon

E-mail address: mna@bau.edu.lb 


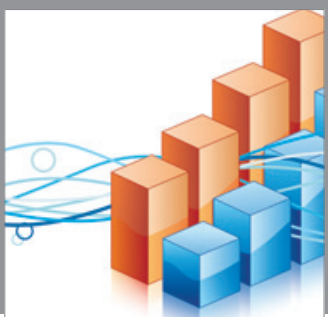

Advances in

Operations Research

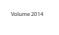

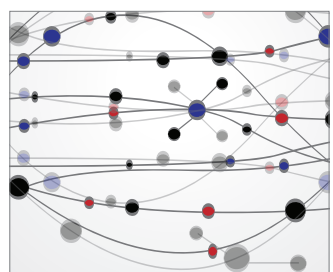

\section{The Scientific} World Journal
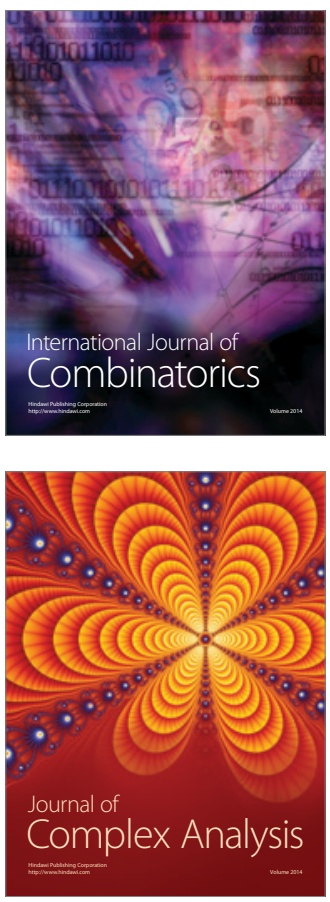

International Journal of

Mathematics and

Mathematical

Sciences
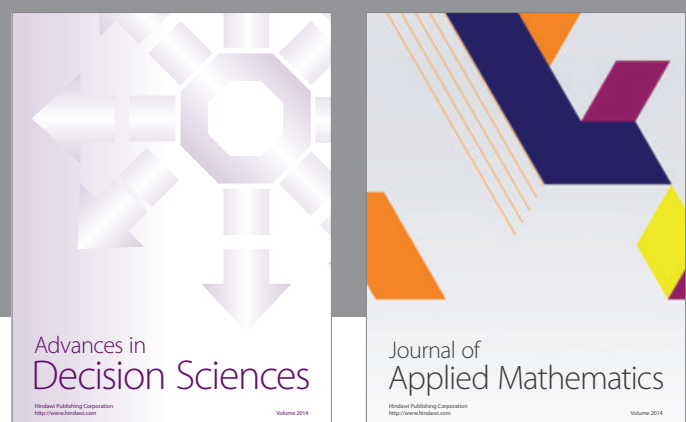

Journal of

Applied Mathematics
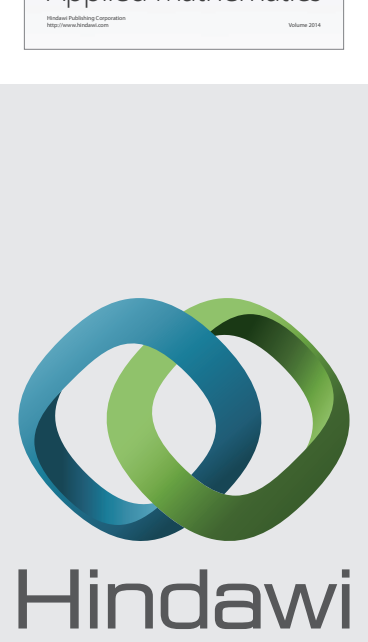

Submit your manuscripts at http://www.hindawi.com
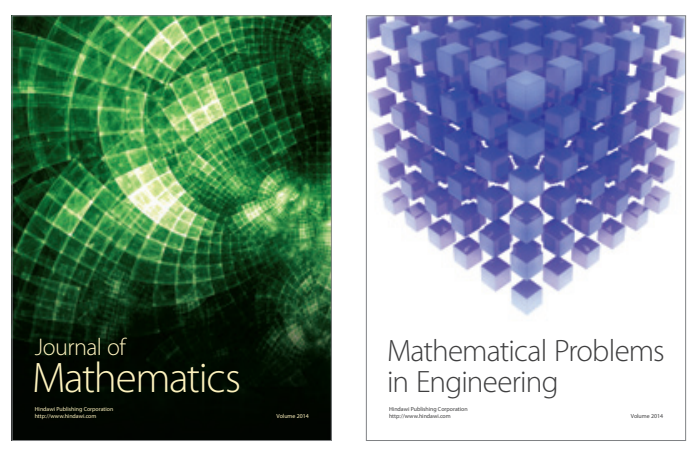

Mathematical Problems in Engineering
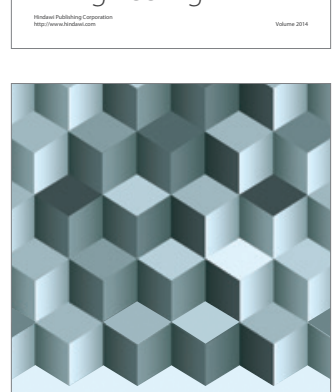

Journal of

Function Spaces
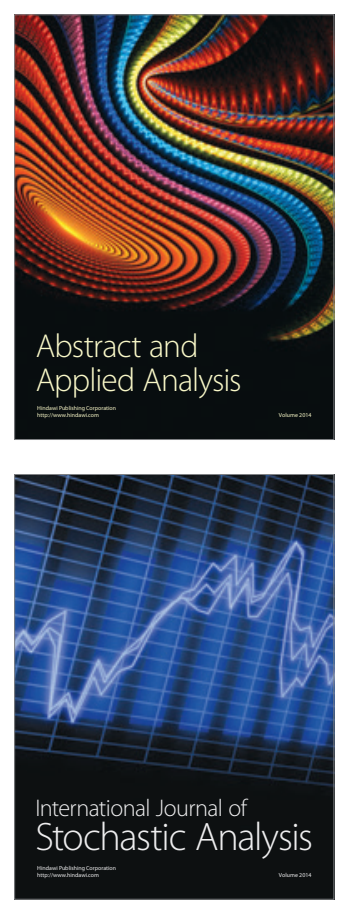

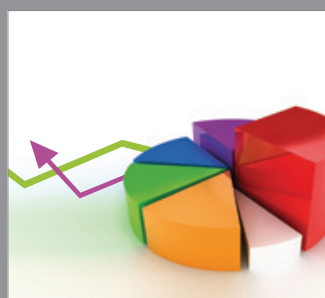

ournal of

Probability and Statistics

Promensencen
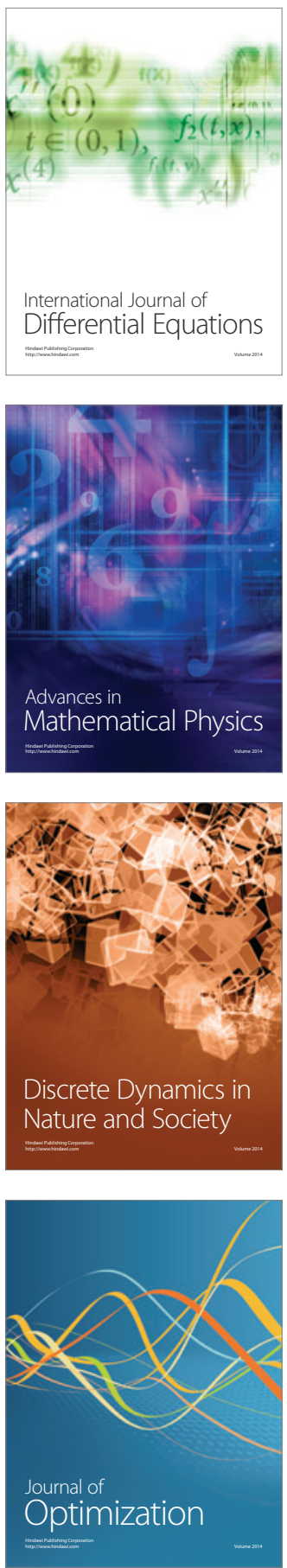